\title{
El diseño de una investigación: el proyecto RAFODIUN
}

\section{The Design of a Research: RAFODIUN Project}

\author{
Julio Barroso Osuna \\ Universidad de Sevilla \\ ibarroso@us.es \\ Julio Cabero Almenara \\ Universidad de Sevilla \\ cabero@us.es
}

Recibido: 12/12/2016

Aceptado: $23 / 12 / 2016$

Publicado: 29/12/2016

\section{RESUMEN}

En el presente artículo, como ejemplo de una investigación presentada en el campo de las ciencias sociales, una investigación realizada alrededor del diseño y producción de objetos de Realidad Aumentada (RA). Una tecnología que pensamos que se está convirtiendo en una tecnología emergente, con grandes posibilidades para su uso en la educación, permitiéndonos la posibilidad de enriquecer la realidad física con información digital en tiempo real. Se incluyen las diferentes fases que hemos tenido en cuenta, así como los principales resultados que se van obteniendo.

\section{PALABRAS CLAVE}

Realidad aumentada; educación; enseñanza superior; investigación educativa

\section{ABSTRACT}

In the present article, as example of an investigation presented in the field of the social sciences, an investigation realized about the design and production of objects of Augmented Reality (AR). A technology that we think that is turning into an emergent technology, with big possibilities for his use into the education, allowing us the possibility of enriching the physical reality with digital real time information. There are included the different phases that we have born in mind, as well as the principal results that are obtained.

\section{KEYWORDS}

Augmented Reality; education; higher education; educational research

\section{CITA RECOMENDADA}

Barroso, J. y Cabero, J. (2016). El diseño de una investigación: el proyecto RAFODIUN. RIITE. Revista Interuniversitaria de Investigación en Tecnología Educativa, 1, 12-25. Doi: http://dx.doi.org/10.6018/riite/2016/277651 


\section{Principales aportaciones del artículo y futuras líneas de investigación:}

- Aporte de trabajos relacionados con la investigación en el campo de la Realidad Aumentada, para de esta forma contribuir al escaso número de experiencias relacionadas con esta temática en el campo educativo.

- Una vez analizado con los estudiantes las valoraciones positivas que han realizado sobre los objetos, y que los perciben como fáciles de utilizar, van a ir en la línea de incorporarlos en situaciones reales de enseñanza-aprendizaje, donde se analicen las actitudes que los objetos desprenden en los estudiantes y su papel en la adquisición de rendimientos por parte de los estudiantes.

\section{INTRODUCCIÓN}

En el presente artículo, hablaremos de las diferentes fases a tener en cuenta en el proceso de elaboración de un proyecto de investigación, así como el avance de alguno de los resultados obtenidos, concretamente nos centraremos en el proyecto titulado "Realidad Aumentada para Aumentar la Formación. Diseño, producción y evaluación de programas de realidad aumentada para la formación universitaria" (EDU-5746-P) (RAFODIUN). Este proyecto lo presentamos a la convocatoria de proyectos de I+D del Ministerio de Economía y Competitividad del Gobierno de España.

Para comenzar decir que toda investigación se convierte en un proceso de indagación, que la profesora Delgado (2014), describió como "un viaje a Itaca", en el cual cada una de las singladuras realizadas durante dicho viaje debe ser justificada. Por lo tanto intentaremos explicar, desde los motivos que nos llevaron a la elección del tema hasta algunos de los resultados que vamos obteniendo, pasando por las diferentes fases del mismo y la metodología utilizada.

\section{LA ELECCIÓN DEL TEMA}

El motivo que nos llevó a trabajar con la "Realidad Aumentada" ("Augmented Reality") (RA), fue el que nos encontramos ante una tecnología de las denominadas emergentes y con verdaderas posibilidades de impactar en el terreno educativo. Este aspecto se pone de manifiesto en los diferentes Informes Horizon (García et al., 2010; Johnson et al., 2016) y el Reporte EduTrend del Observatorio del Tecnológico de Monterrey (Tecnológico de Monterrey, 2015), estos estudios ponen de manifiesto que nos encontramos ante una tecnología que tendrá una fuerte penetración en las instancias educativas en un horizonte de 3 a 5 años (Bacca, Baldiris, Fabregat, Graf, \& Kinshuk, 2014; Tecnológico de Monterrey, 2015; Johnson \& Adams, 2016; Cabero \& Barroso, 2016; Cabero \& García, 2016). Por su parte, la compañía "Gardner Research" (http://www.gartner.com/technology/home.jsp), líder mundial en investigación y asesoramiento en Tecnologías de la Información y Comunicación (TIC), la identifica como una de las diez tecnologías que tendría más impacto en los próximos años, con una previsión de uso en el año 2014, del orden del $30 \%$ de los usuarios que dispongan de dispositivos móviles. Previsión que ha ido creciendo, como pone de manifiesto que al buscar por el término "realidad aumentada" el buscador google nos ofrece "aproximadamente 1.510 .000 resultados en 0,71 segundos" y si ubicamos "augmented reality", el número de documentos se eleva a 19.300.000 en una búsqueda de 0,66 segundos.

Si buscamos una primera aproximación al término RA, podemos decir que se trata de una tecnología que permite la combinación de información digital e información física en tiempo real por medio de distintos soportes tecnológicos como por ejemplo las tablets o los smartphones, para crear con ello una nueva realidad enriquecida. Su significación para la formación viene determinada, por una parte por sus características específicas, como son: ser una realidad mixta, la posibilidad que ofrece para integrar en tipo real tanto diferentes capas de información 
como información en diferentes tipos de formatos (textos, url, vídeos,...), es una tecnología interactiva, su facilidad de manejo, y que mediante su utilización enriquecemos o alteramos la información de la realidad añadiéndole información adicional (Cabero y García, 2016). Y por otra, porque los dispositivos que se suelen utilizar para su observación, como son los smartphones, son tecnologías de las que disponen con facilidad los alumnos universitarios.

Su utilización en la formación, como señalan diferentes autores $(\mathrm{Wu}$, Wen-Yu, Chang, \& Liang, 2013; Carozza, Tingdahl, Bosché \& Gool, 2014; Cubillo, Martín, Castro, \& Colmenar, 2014; Jeřábek, Rambousek. \& Wildová; 2014; Barba, Yasaca, \& Manosalvas, 2015; Jamali, Fairuz, Wai, \& Oskam, 2015; Fonseca, Redondo, \& Valls, 2015; Han, Jo, Hyun, \& So, 2015; Prendes, 2015; Cabero \& Barroso, 2016; Santos, Wolde, Taketomi, Yamamoto, Rodrigo, Sandor, \& Kato, 2016), nos permite diferentes posibilidades como son: a) eliminar información que pueda entorpecer la captación de la información significativa por el estudiante; b) aumentar o enriquecer la información de la realidad para hacerla más comprensible al estudiante; c) poder observar un objeto desde diferentes puntos de vista seleccionando el estudiante el momento y posición de observación; d) potencia el aprendizaje ubicuo; e) crear escenarios "artificiales" seguros para los estudiantes como pueden ser laboratorios o simuladores; f) enriquecer los materiales impresos para los estudiantes con información adicional en diferentes soportes; g) y convertir a los alumnos en "proconsumidores" de objetos de aprendizaje en formato RA. A ellas debemos incorporarles que puede ser utilizada en diferentes disciplinas y en distintos niveles educativos (De Pedro Carracedo \& Méndez, 2012; Bressler \& Bodzin, 2013; Prendes, 2015; Cabero \& García, 2016), aunque se debe reconocer que es en la formación universitaria donde se está utilizando con mayor frecuencia (Lin, Been-Lirn, Li, Wang, \& Tsai, 2013).

\section{EL PROYECTO RAFODIUN}

Con la intención de profundizar en las posibilidades educativas de la RA en el contexto universitario, nos planteamos los siguientes objetivos:

- Evaluar las posibilidades y potencialidades que ofrecen diferentes software utilizados para la creación de entornos tecnológicos bajo la arquitectura de la RA para ser utilizados en contextos formativos universitarios.

- Analizar las posibilidades que los diferentes tipos de dispositivos de RA ofrecen para su aplicación en contextos de enseñanza universitaria.

- Diseñar y producir distintos contenidos en formato RA para ser aplicados en contextos de formación universitaria en distintas áreas curriculares, y evaluar sus posibilidades de cara al rendimiento de los alumnos.

- Conocer el grado de motivación y nivel de satisfacción que despierta en los estudiantes universitarios el hecho de participar en experiencias formativas apoyadas en RA.

- Crear un entorno formativo bajo la arquitectura de la RA, en formato libro electrónico, para la capacitación del profesorado universitario en el diseño, producción y utilización educativa de la RA.

- Poner en acción y validar el entorno producido para la capacitación del profesorado universitario en el diseño, producción y utilización educativa de la RA.

- Conocer las posibilidades educativas que permite el que el alumno se convierta en como productor de experiencias formativas apoyadas en la RA.

- Indagar sobre las dificultades técnicas, curriculares y organizativas que pudieran tener la RA para ser aplicada a los contextos de formación universitaria.

- Y crear una comunidad virtual formada por profesorado universitario preocupado por la utilización educativa de la RA. 
Teniendo en cuenta los objetivos planteados y que como hemos comentado, pretendemos analizar las posibilidades educativas que para contextos de formación universitaria puede tener la RA. Lo siguiente que tenemos que planificar es las diferentes perspectivas que tenemos que tener en cuenta en nuestro estudio. En este caso, tendremos en cuenta aspectos tanto tecnológicas-instrumentales, como educativas, de diseño de entornos formativos, hasta la referida a la formación y el perfeccionamiento del profesorado, y al alumno como productor de contenidos en RA.

El alcance de los diferentes objetivos se llevará a cabo mediante diferentes tipos de técnicas y estrategias usualmente utilizadas en la investigación en el terreno de la Tecnología Educativa (Barroso y Cabero, 2010), y a través de diferentes fases:

Primera Fase: Evaluación y análisis del software de producción de la RA y de los diferentes tipos de dispositivos.

En esta primera fase se realizarán distintas actividades encaminadas a evaluar los diferentes programas de software informáticos existentes en el mercado para la producción de entornos de RA, y así como la evaluación de los diversos tipos de dispositivos que se pueden utilizar para la producción de entornos en RA. Con ello pretendemos dar respuesta a los objetivos 1 y 2 señalados anteriormente.

Etapa 1.- Evaluación del software informático de producción de entornos RA. Se realizarán las siguientes actividades:

- Revisión e identificación de los principales programas informáticos que existen en el mercado para la producción de contenidos en RA.

- Configuración de una lista de programas con sus direcciones web, e identificación de versiones de prueba de los programas de producción de RA.

- Creación de una lista de chequeo para la evaluación de los programas identificados.

- Elaboración de la versión en Internet del instrumento.

- Evaluación del software mediante la aplicación de la técnica del juicio de experto.

Etapa 2.- Análisis de las posibilidades que los diferentes tipos de dispositivos de RA ofrecen para su aplicación en contextos de enseñanza universitaria, para ello se realizarán las siguientes actividades:

- Configuración de grupos de discusión.

- Realización de los grupos de discusión, que se realizarán de acuerdo a la siguiente estructura: introducción por el moderador de los objetivos que se persiguen con la actividad y de los objetivos que perseguimos con nuestro trabajo, presentación de las personas que conforman el grupo de discusión, exposición de los diferentes dispositivos, aclaración de dudas sobre los objetivos y los dispositivos utilizados en la RA, y realización de la sesión.

Etapa 3.- Crear una comunidad virtual de profesorado universitario preocupado por la utilización educativa de la RA.

Segunda Fase: Diseñar y producir contenidos formativos en formato RA para ser aplicados en contextos de formación universitaria.

Para la producción de los contenidos en diferentes formatos de RA, se seguirá el siguiente procedimiento: Selección de los contenidos, producción de los contenidos en RA, producción técnica a través de diferentes herramientas y evaluación mediante un grupo de expertos y diferentes estudios pilotos. 
Por lo que se refiere a los estudios pilotos que se llevarán a cabo, queremos saber si los alumnos tras interaccionar con los contenidos elaborados en RA, aprendían la información y conceptos que se presentaban en los mismos. Entendiendo en este caso por aprender la capacidad de recordar, comprender, y aplicar la información presentada en los contenidos, y entendiendo por estas categorías lo especificado en la taxonomía de Bloom para la era digital (Chursches, 2008). El instrumento a aplicar será del tipo de elección múltiple, y se administrará en la modalidad de pretets y postests.

Para el diagnóstico del nivel de aceptación y de satisfacción de la tecnología de la RA por el estudiante utilizaremos la adaptación realizada por Wojciechowski y Cellary (2013) del cuestionario "Modelo de Aceptación de Tecnología" (TAM) propuesto inicialmente por Davies y otros (1992) instrumento ampliamente utilizado en los estudios de aceptación de tecnología (Teo, Beng, Chai, \& Wong, 2009; Sun y Cheng, 2009; Teo y Noyes, 2011).

En el modelo de TAM, la aceptación de la tecnología está representado por intención de utilizarla, que está determinada por la actitud del usuario hacia su uso y la utilidad que percibe de ella. Como señalan los autores del instrumento la actitud hacia el uso de una tecnología está determinada por la percepción de la utilidad y facilidad de uso del sistema de los usuarios, está determinada por la facilidad de uso percibida. Además, la utilidad percibida y la facilidad de uso percibida, puede estar afectada por diversas variables externas, como son las características del usuario, y las características de la tecnología y el entorno en el que se utiliza el sistema.

El diagnóstico de la motivación que había despertado la participación en la experiencia de RA por los alumnos, lo efectuaremos mediante la "Encuesta de Motivación Materiales de Instrucción" (IMMS) construida por Keller (2010) y utilizada por Di Serio y otros, (2013) en su investigación sobre RA, y que considera cuatro factores de motivación: atención, interés, confianza y satisfacción.

Tercera Fase: crear un entorno formativo bajo la arquitectura de la RA en formato libro electrónico, para la capacitación del profesorado universitario en el diseño, producción y utilización educativa de la RA.

En este caso se seguirá un procedimiento de construcción y evaluación del material elaborado muy parecido al señalado anteriormente.

Cuarta Fase: Conocer las posibilidades educativas que nos ofrece el alumno como productor de experiencias formativas apoyadas en la RA.

En 1985 miembros del equipo de investigación realizaron la investigación "Evaluación de los usos del vídeo en la enseñanza" financiada por el Ministerio de Educación y Ciencia (López-Arenas y Cabero, 1990), con él pretendíamos ver las posibilidades educativas que tendría diversas formas de utilizar el vídeo y una de ellas era su utilización como instrumento de conocimiento por parte de los alumnos; es decir, la posibilidad que los alumnos se conviertan en productores de videomensajes y analicen de esta forma la realidad. $Y$ ello es precisamente lo que perseguimos con esta fase de la investigación que los alumnos se conviertas en productores de materiales educativos con estructura de RA. Con ello pretendemos alcanzar el objetivo 7 señalado anteriormente.

Su ejecución se llevará a cabo a través de diferentes etapas, que van desde selección de los grupos, formación de los grupos, trabajo en grupo por los alumnos para la elaboración de contenidos en formato RA, y evaluación de la experiencia. 


\section{AVANCES PRODUCIDOS EN EL PROYECTO Y PRIMEROS RESULTADOS}

Hasta el momento a falta de un año para la finalización del proyecto, se han venido realizando la mayoría de las acciones. En primer lugar, se ha creado un sitio web (Figura 1) para presentar en el mismo las diferentes aportaciones que se van realizando, así como los diferentes objetos de aprendizaje producidos para el proyecto (http://intra.sav.us.es/proyectorafodiun/), en dicho lugar se puede acceder a diferentes informaciones como son los participantes del proyecto, y las diferentes actividades que se están llevando respecto al mismo.
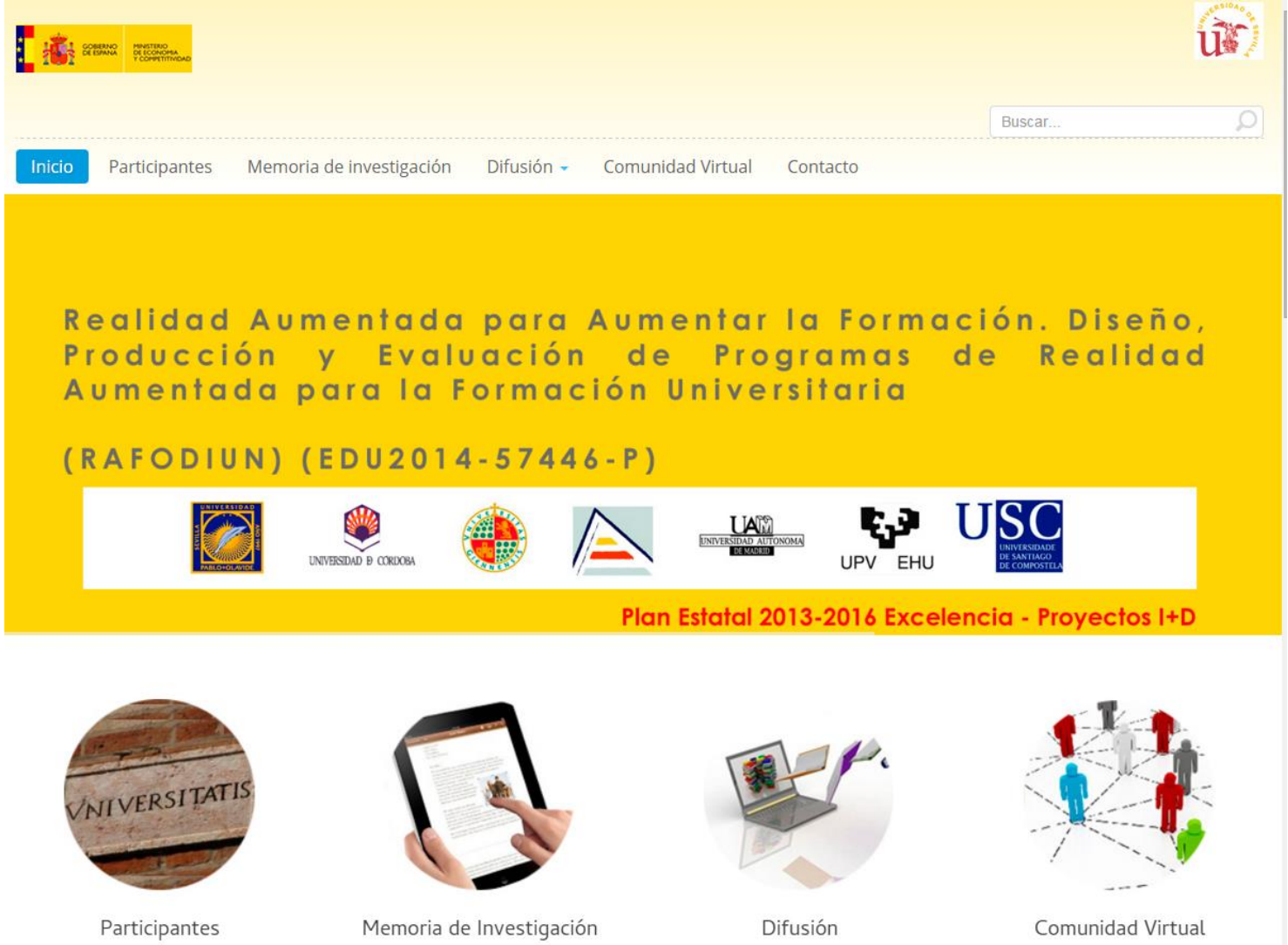

Figura 1. Sitio web del proyecto

Uno de los objetivos más avanzados del proyecto es el referido a la creación de la comunidad virtual (Figura 2). La comunidad virtual RAFODIUN se puso en funcionamiento el 21 de junio del 2015 (https://plus.google.com/u/0/communities/102143147822806126247), y se elaboró dentro del programa Google+. En la actualidad cuenta con 513 miembros de diferentes países (Uruguay, Costa Rica, Colombia, República Dominicana, Francia, México, Venezuela, Brasil, Bolivia, Chile, Puerto Rico, Cuba y España, Siendo los más numerosos los españoles que ocupan cerca del $75 \%$. En la misma existe un número equilibrado de hombres y mujeres, aunque son los hombres los que han realizado el mayor número de aportaciones. En ella aparecen fenómenos tradicionales en las comunidades virtuales, donde pocos sujetos aglutinan la gran mayoría de las aportaciones; como podemos observar en la fig. ํㅡㄹ 2, donde se puede observar que en el centro aparecen los sujetos que más han participado, análisis efectuado mediante el programa Pajeck. 


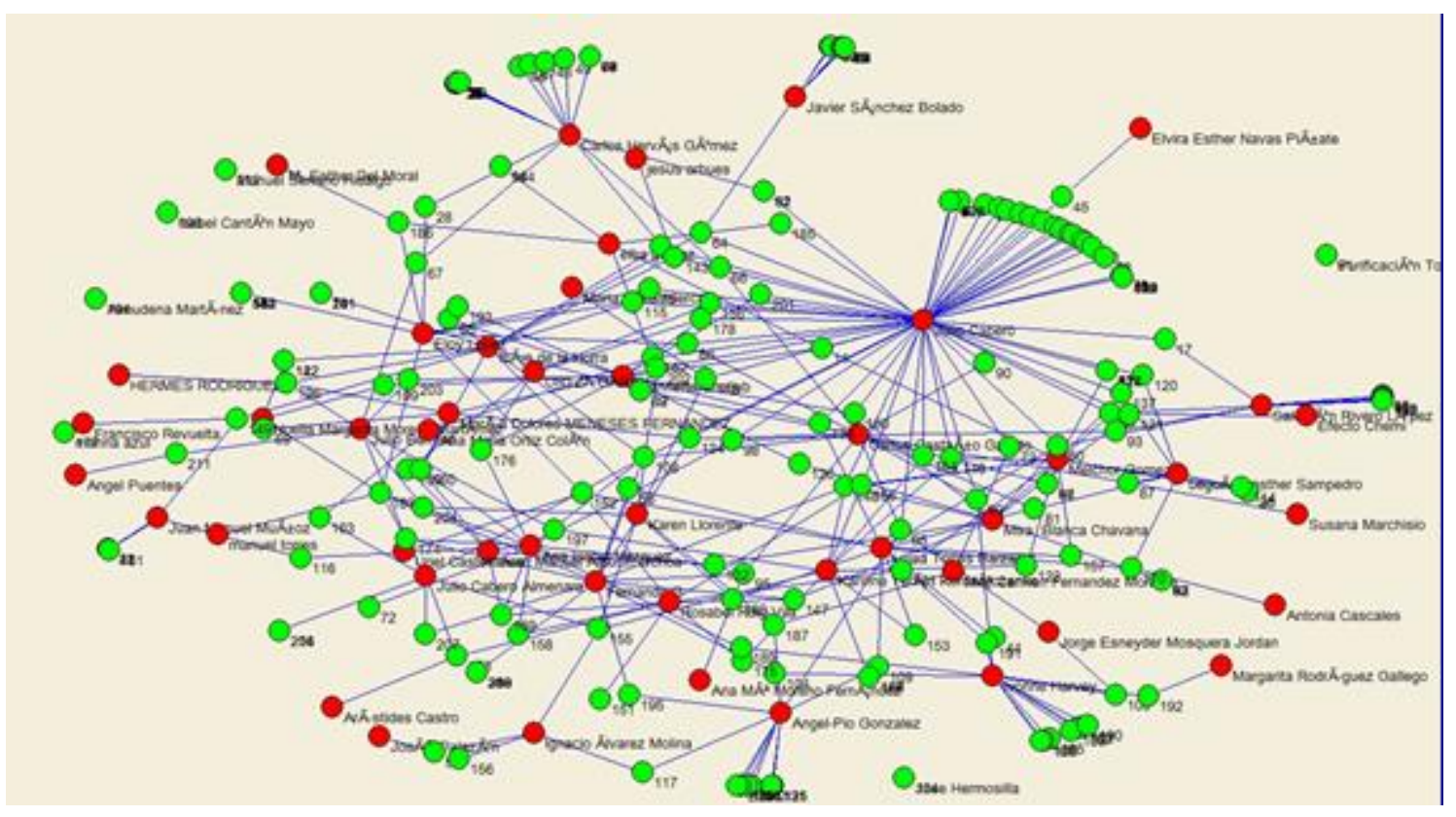

Figura 2. Análisis de la Comunidad virtual RAFODIUN.

Y por aportar otro dato, indicar que a comienzo de diciembre del 2016 se habían producido 415 post y en 214 de ellos, se ha producido algún tipo de participación de algún miembro de la comunidad. Los que suponía que en el $51,57 \%$ de los post se ha producido algún tipo de respuesta escrita a los presentado por el autor. En 87 de los 214 ha existido algún tipo de respuesta del autor del post a los comentarios realizados por algún miembro de la comunidad; lo que supone que el $40,65 \%$ de los post en los que ha existido algún tipo de participación escrita, ha respondido el autor del post a los comentarios realizados por algún miembro de la comunidad.

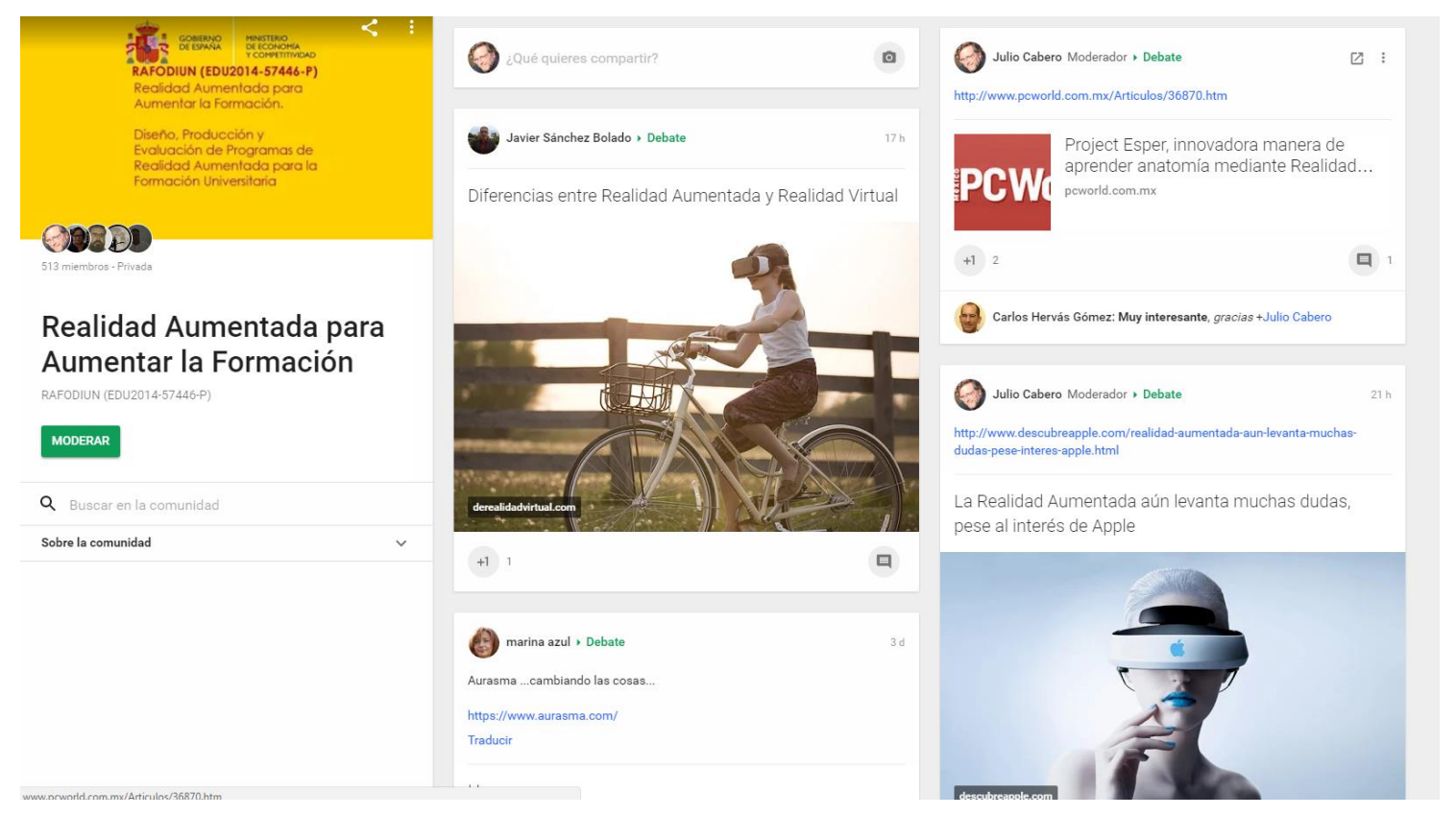

Figura 3. Comunidad virtual RAFODIUN 
También se han venido produciendo los diferentes objetos de RA, con los que llevaremos a cabo la segunda fase de nuestro estudio. Concretamente se han producido objetos en el campo de las ciencias de la educación y para la enseñanza de la medicina. Uno de los objetos es el referido al "Diseño, producción y evaluación de Tecnologías de la Información y Comunicación para la enseñanza", el lanzador del mismo puede observarse en la figura 4.

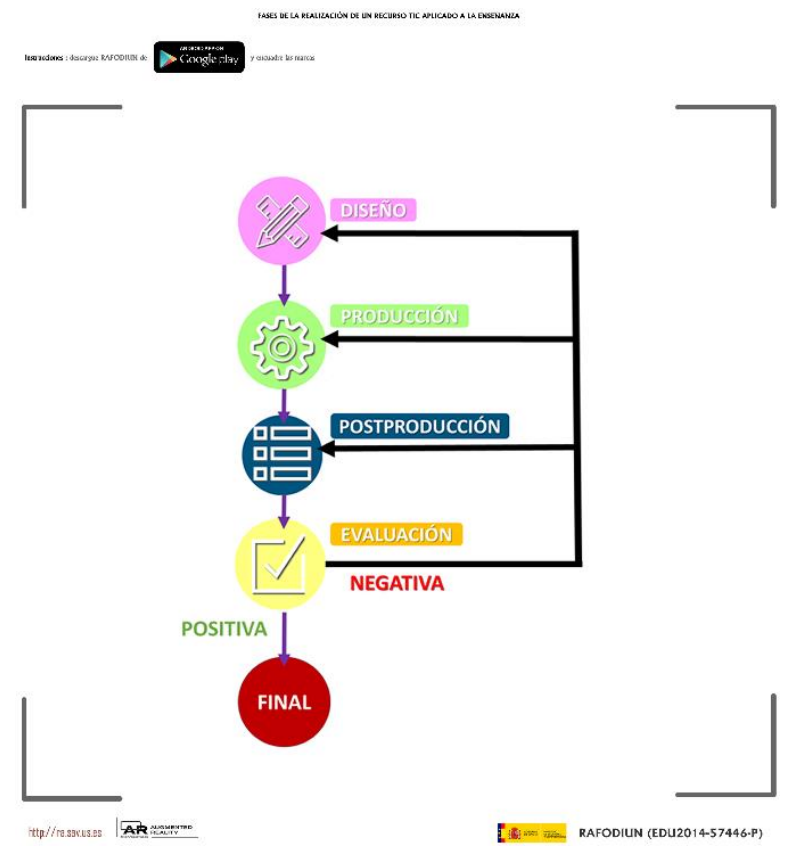

Figura 4. Lanzador del objeto RA producido: Diseño, producción y evaluación de Tecnologías de la Información y Comunicación para la enseñanza.

A modo de ejemplo en la tabla 1 , se presenta el guión del objeto producido y la diversidad de recursos que incorpora.

\begin{tabular}{|c|c|c|}
\hline Secuencia & Tipo de recurso & Descripción del recurso \\
\hline 1 & $\begin{array}{l}\text { Clip de video. Producción } \\
\text { propia }\end{array}$ & $\begin{array}{l}\begin{array}{l}\text { Explica de forma general la significación del diseño. Tipos } \\
\text { de diseño. }\end{array}\end{array}$ \\
\hline 2 & $\begin{array}{l}\text { Persona encima de un } \\
\text { esquema (Fernando- } \\
\text { símbolos químicos) }\end{array}$ & $\begin{array}{c}\text { La persona indicara que todo diseño de una tecnología } \\
\text { pasa por cuatro etapas: Diseño, producción, } \\
\text { postproducción y evaluación. Si la evaluación es positiva } \\
\text { termina el ciclo y en caso contrario hay que volver a } \\
\text { revisarlo. }\end{array}$ \\
\hline 3 & Polimedia & $\begin{array}{c}\text { Contar la primera fase. Diseño. Esta fase termina con la } \\
\text { elaboración de un guión. }\end{array}$ \\
\hline 3.1 & $\begin{array}{c}\text { Web en la que puedan } \\
\text { observar diferentes } \\
\text { modelos de guión }\end{array}$ & Modelos de guión \\
\hline 4 & $\begin{array}{l}\text { Clip de video de } \\
\text { producción propia en el } \\
\text { que podemos ver } \\
\text { diferentes momentos en el } \\
\text { proceso de producción. }\end{array}$ & Explicar la fase 2 y la 3 \\
\hline 5 & Polimedia & $\begin{array}{l}\text { Evaluación. Explica las diferentes estrategias y tipos de } \\
\text { evaluación. }\end{array}$ \\
\hline
\end{tabular}




\begin{tabular}{|c|c|c|}
\hline 5.1 & Animación 3D & $\begin{array}{r}\text { Explicamos las ventajas e inconvenientes de cada una de } \\
\text { las estrategias, a nivel de síntesis }\end{array}$ \\
\hline 5.2 & $\begin{array}{c}\text { Listado interactivo que nos } \\
\text { ofrezca ejemplos }\end{array}$ & Explicar las diferentes técnicas de evaluación. \\
\hline 5.3 & Pdf & Incluya las diferentes dimensiones \\
\hline 6 & Web & Contenido bibliografía y "para saber más" \\
\hline 7 & PDF & $\begin{array}{r}\text { Ejercicio de evaluación. 10 preguntas tipo test de } \\
\text { diferentes modalidades. }\end{array}$ \\
\hline
\end{tabular}

Tabla 1. Guión del objeto producido en RA

En la tabla 2, se presenta una relación de algunos de los programas de software informáticos utilizados para la producción de los diferentes objetos.

\begin{tabular}{|c|c|c|}
\hline SOFTWARE & PARA QUÉ SE UTILIZA & $\begin{array}{l}\text { OBJETO PRODUCIDO EN EL } \\
\text { QUE HA SIDO UTILIZADO }\end{array}$ \\
\hline Blender & $\begin{array}{l}\text { Modelado y animación 3d. } \\
\text { Texture baking }\end{array}$ & Web2.0 \\
\hline 3dmax & $\begin{array}{l}\text { Modelado y animación 3d. } \\
\text { Texture baking. }\end{array}$ & \\
\hline Maya & Modelado y animación 3d & \\
\hline Rhino & $\begin{array}{l}\text { Modelado y animación 3d. } \\
\text { Reducción de polígonos }\end{array}$ & \\
\hline Metaio Creator & $\begin{array}{l}\text { Programación realidad } \\
\text { aumentada. }\end{array}$ & $\begin{array}{c}\text { Viea } \\
\text { Diseño } \\
\text { Web } 2.0\end{array}$ \\
\hline Metaio SDK & Kit de desarrollo de software RA & $\begin{array}{c}\text { Viea } \\
\text { Diseño } \\
\text { Web } 2.0\end{array}$ \\
\hline Eclipse & $\begin{array}{l}\text { Entorno de desarrollo Java. } \\
\text { Exportación apk para android. }\end{array}$ & $\begin{array}{c}\text { Viea } \\
\text { Diseño }\end{array}$ \\
\hline Android Studio & $\begin{array}{l}\text { Entorno de desarrollo Java. } \\
\text { Exportación apk para android. }\end{array}$ & Web 2.0 \\
\hline Xcode & $\begin{array}{c}\text { Entorno de desarrollo Java. } \\
\text { Exportación .ipa para IOS.Subida } \\
\text { a la app store. }\end{array}$ & $\begin{array}{c}\text { Viea } \\
\text { Diseño } \\
\text { Web } 2.0\end{array}$ \\
\hline Adobe After effects & $\begin{array}{l}\text { Postproducción de vídeo y } \\
\text { sonido. Chroma, Rotobrush, Key } \\
\text { Light. }\end{array}$ & $\begin{array}{c}\text { Viea } \\
\text { Diseño } \\
\text { Web } 2.0\end{array}$ \\
\hline Adobe Photoshop & $\begin{array}{l}\text { Postproducción de imagen. } \\
\text { Grafismos. Photomerge. } \\
\text { Texturizado 3d. }\end{array}$ & $\begin{array}{c}\text { Viea } \\
\text { Diseño } \\
\text { Web } 2.0\end{array}$ \\
\hline
\end{tabular}




\begin{tabular}{|c|c|c|}
\hline Macromedia Fireworks & $\begin{array}{l}\text { Postproducción de imagen. } \\
\text { Grafismos. }\end{array}$ & $\begin{array}{c}\text { Viea } \\
\text { Diseño } \\
\text { Web } 2.0\end{array}$ \\
\hline Unity 5 & 3d. Entorno de desarrollo & \\
\hline Ffmpeg & $\begin{array}{c}\text { Programación sobre el codec } \\
\text { para exportación vídeos 3g2. } \\
\text { Augment----RA }\end{array}$ & $\begin{array}{c}\text { Viea } \\
\text { Diseño } \\
\text { Web } 2.0\end{array}$ \\
\hline MS Powerpoint & $\begin{array}{l}\text { Botones en formato vídeo con } \\
\text { efectos de transición }\end{array}$ & \\
\hline Notepad ++ & $\begin{array}{l}\text { Editor profesional de texto para } \\
\text { retoque de código. }\end{array}$ & $\begin{array}{c}\text { Viea } \\
\text { Diseño } \\
\text { Web } 2.0\end{array}$ \\
\hline Metaio toolbox & $\begin{array}{l}\text { Extracción marcador basado en } \\
\text { 3d }\end{array}$ & \\
\hline Autocatch123D & Fotogrametría & \\
\hline Artec Studio & Escaneo 3d & \\
\hline Astrum & Creador de instalador windows & $\begin{array}{c}\text { Viea } \\
\text { Diseño } \\
\text { Web } 2.0\end{array}$ \\
\hline
\end{tabular}

Tabla 2. Software utilizado para la producción de los objetos

Los estudios realizados hasta el momento han puesto de manifiesto diferentes conclusiones:

- Los instrumentos de diagnóstico del IMMS de Keller (2010) como el TAM de Davis (1989), se presentan como buenos predictores para explicar la motivación (atención, confianza, relevancia y satisfacción) y grado de adopción de la tecnología de la RA. Al mismo tiempo los instrumentos de diagnóstico utilizados se han presentado fiables, mostrando índices de fiabilidad similares a los alcanzados por otros autores en diferentes investigación, y ello ocurría tanto con el instrumento IMMS (Huang, Huang, \& Tschopp, 2010; Keller, 2010; Che, 2012; Di Serio, Blanca, \& Delgado, 2013; Yufeng \& Yamanaka, 2013; Proske, Roscoe, \& McNamara, 2014) como con el TAM (Yong, Rivas, \& Chaparro, 2010; Park, Nam, \& Cha, 2012; Ho, Hung, \& Chen, 2013; Cheng, Lou, Kuo, \& Shih, 2013; Tarhini, Hone, \& Liu, 2014).

- Los objetos de aprendizaje en RA se presentan como materiales educativos de utilidad para el aprendizaje de los alumnos de Medicina, aspecto en el que coincidimos con otros autores (Yeo et al., 2011; Wu, Wen-Yu, Chang \& Liang, 2013; Bower et al., 2014).

- Algunos estudiantes han encontrado dificultad a la hora de interaccionar con estos objetos, ello posiblemente se deba a su falta de experiencia y novedad de la tecnología, hecho que creemos que desaparecerá con un uso más habitual.

- Su incorporación a la enseñanza es fácil, pues casi todos los alumnos tienen dispositivos móviles y saben descargarse app.

- El género del estudiante no determina el grado de aceptación que tienen de la RA, lo que coincide con los hallazgos de no relación entre género y tecnologías de otros autores (Cabero, Leal, Andrés \& Llorente, 2009; Bullón et al., 2009). 
En la actualidad nos encontramos en la fase de análisis de los datos alcanzados en los estudios producidos para analizar las posibilidades que ofrece la producción de objetos de aprendizaje en RA. Nuestra impresión es que la misma despierta verdadero interés en los estudiantes y que los mismos son capaces de producir objetos en este tipo de tecnología, sobre todo bajo la modalidad que hemos denominado como "apuntes enriquecidos".

El reto que nos queda es el de elaborar un curso de formación del profesorado en formato $R A$, estando en la actualidad en la fase de determinación de los contenidos que deben incorporarse.

\section{REFERENCIAS BIBLIOGRÁFICAS}

Bacca, J., Baldiris, S., Fabregat, R., Graf, S. y Kinshuk. (2014). Augmented reality trends in education: a systematic review of research and applications. Educational Technology \& Society, $17(4)$, pp. 133-149.

Barba, R., Yasaca, S. y Manosalvas, C. (2015). Impacto de la realidad aumentada móvil en el proceso enseñanza-aprendizaje de estudiantes universitarios del área de medicina. Investigar con y para la Sociedad, 3, Cádiz: Bubok Publishing, SL, pp. 1421-1.429.

Barroso, J. y Cabero, J. (2010). La investigación educativa en TIC. Visiones prácticas. Madrid: Síntesis.

Bower, M., Howe, C., McCredie, N., Robinson, A. y Grover, D. (2014). Augmented Reality in education - cases, places and potentials. Educational Media International, 51(1), pp. 1-15.

Bressler, D. M. y Bodzin, A. M. (2013). A mixed methods assessment of students' flow experiences during a mobile augmented reality science game. Journal of Computer Assisted Learning, 29 (6), pp. 505-517.

Bullón, P., Cabero, J., Llorente, M.C., Machuca, M.C., Machuca, G. y Marín, V. (2009). Competencias tecnológicas del profesorado de la Facultad de Odontología de la Universidad de Sevilla. Sevilla: GID.

Cabero, J. y Barroso, J. (2016). The educational possibilities of Augmented Reality. NAER. New Approaches in Educational Research, 5 (1), pp. 44-50.

Cabero, J. y García, F. (coords.) (2016). Realidad aumentada. Tecnología para la formación. Madrid: Síntesis.

Cabero, J., Leal, F., Andrés, F. y Llorente, M.C. (2009). La alfabetización digital de los alumnos universitarios mexicanos. Enseñanza \& Teaching, 27 (1), pp. 41-59.

Carozza, L., Tingdahi, D., Bosché, F. y Gool, L. (2012). Markerless vision-based augmented reality for urban planning. Computer-Aided Civil and Infrastructure Engineering, 00, pp. 1-16.

Che, Y. (2012). A study of learning effects on e-learning with interactive thematic video. Journal Educational Computing Research, 47(3), pp. 279-292. doi: http://dx.doi.org/10.2190/EC.47.3.c

Cheng, Y., Lou, S., Kuo, S, y Shih, R. (2013). Investigating elementary school students' technology acceptance by applying digital game-based learning to environmental education. Australasian Journal of Educational Technology, 29(1) pp. 96-110. 
Chursches, A. (2008). Taxonomía de Bloom para la era digital. Eduteka. Recuperado de http://www.eduteka.org/TaxonomiaBloomDigital.php-

Cubillo, J., Martín, S., Castro, M. y Colmenar, A. (2014). Recursos digitales autónomos mediante realidad aumentada. RIED, 17 (2), pp. 241-274.

Davis, F. (1989). Perceived usefulness, perceived ease of use, and user acceptance of information technology. MIS Quarterly 13 (3) pp. 319-340, doi: 10.2307/249008

Delgado, C. (2014). Viajando a Ítaca por mares cuantitativos. Manual de ruta para investigar en grado y postgrado. Salamanca: Amarú.

Di Serio, A., Blanca, M. y Delgado, C. (2013). Impact of an augmented reality system on students' motivation for a visual art course. Computers \& Education, 68, pp. 586-596. doi:10.1016/j.compedu.2012.03.002

Fonseca, D., Redondo, E. y Valls, F. (2016). Motivación y mejora académica utilizando realidad aumentada para el estudio de modelos tridimensionales arquitectónicos. Education in the Knowledge Society (EKS), 17 (1), pp. 45-64.

García, I., Peña, I., Jhonson, L., Smith, R., Levine, A. y Haywood, K. (2010). Informe Horizon: Edición Iberoamericana 2010. Austin, Texas: The New Media Consortium.

Han, J., Jo, M., Hyun, E. y So, H. (2015). Examining young children's perception toward augmented reality-infused dramatic play. Education Technology Research Development, 63, pp. 455-474. Doi: 10.1007/s11423-015-9374-9

Ho, L-H., Hung, Ch-L. y Chen, H-Ch. (2013). Using theoretical models to examine the acceptance behavior of mobile phone messaging to enhance parent-teacher interaction. Computers \& Education, 61, pp. 105-114. doi: http://dx.doi.org/10.1016/i.compedu.2012.09.009

Huang, W., Huang, W. y Tschopp, J. (2010). Sustaining iterative game playing processes in DGBL: The relationship between motivational processing and outcome processing. Computers \& Education, 55, pp. 789-797. doi: 10.1016/j.compedu.2010.03.011

Jamali, S., Fairuz, M., Wai, K. y Oskam, C. (2015). Utilising mobile-augmented reality for learning human anatomy. Procedia-Social and Behavioral Sciences, 197, pp. 659-668.

Jeřábek, T., Rambousek, V. y Wildová, R. (2014). Specifics of visual perception of the augmented reality in the context of education. Procedia-Social and Behavioral Sciences, 159, págs. 598-604. Doi: 10.1016/j.sbspro.2014.12.432

Johnson, L., Adams Becker, S., Cummins, M., Estrada, V., Freeman, A., and Hall, C. (2016). NMC Horizon Report: 2016 Higher Education Edition. Austin, Texas: The New Media Consortium.

Keller, J. M. (2010). Motivational design for learning and performance. New York: Springer Science+Business. 
Lin, T., Been-Lirn, H., Li, N., Wang, H. y Tsai, Ch. (2013). An investigation of learners' collaborative knowledge construction performances and behavior patterns in an augmented reality simulation system. Computers \& Education, 68, pp. 314-321.

Park, S., Nam, M-W-y Cha, S-B. (2012). University students' behavioral intention to use mobile learning: Evaluating the technology acceptance model. British Journal of Educational Technology, 43, 4, pp. 592-605.

Pedro Carracedo, J. de y Méndez, C. L. M. (2012). Realidad aumentada: una alternativa metodológica en la educación primaria nicaragüense. IEEE-RITA, 7, pp.102-108.

Prendes, C. (2015). Realidad aumentada y educación: análisis de experiencias prácticas. PixelBit. Revista de Medios y Educación, 46, pp. 187-203.

Proske, A., Roscoe, R. y McNamara, D. (2014). Game-based practice versus traditional practice in computer-based writing strategy training: effects on motivation and achievement. Education Technology Research Development, 62:481-505. doi: 10.1007/s11423-014-93492

Santos, M., Wolde, A., Taketomi, T., Yamamoto, G., Rodrigo, M., Sandor, C. y Kato, H. (2016). Augmented reality as multimedia: the case for situated vocabulary learning. Research and Practice in Techology Enhanced Learning, 11 (4), pp. 1-23.

Sun, H.-M. y Cheng, W.-L. (2009). The input-interface of webcam applied in 3D virtual reality system. Computers \& Education, 53 (4), pp. 1.231-1.240.

Tarhini, A., Hone, K. y Liu, X. (2014). Measuring the moderating effect of gender and age on elearning acceptance in england: a structural equation modeling approach for an extended technology acceptance model. Journal educational computing research, 51(2), pp. 163-184.

Tecnológico de Monterrey (2015). Reporte EduTrends. Radar de Innovación Educativa 2015. Monterrey: Tecnológico de Monterrey.

Teo, T., Beng, Ch., Chai, Ch. y Wong S. (2009). Assessing the intention to use technology among pre-service teachers in Singapore and Malaysia: A multigroup invariance analysis of the Technology Acceptance Model (TAM). Computers \& Education, 53, pp. 1000-1009.

Teo, T., y Noyes, J. (2011). An assessment of the influence of perceived enjoyment and attitude on the intention to use technology among pre-service teachers: a structural equation modeling approach. Computers \& Education, 57(2), pp. 1645-1653.

Wojciechowski, R. y Cellary, W. (2013). Evaluation of learners' attitude toward learning in ARIES augmented reality environments. Computers \& Education, 68, pp. 570-585.

Wu, H.-S., Wen-Yu, S., Chang, H.-Y. y Liang, J. (2013). Current status, opportunities and challenges of augmented reality in education. Computers \& Education, 62, pp. 41-49. http://dx.doi.org/10.1016/j.compedu.2012.10.024

Yeo, C. T., et al. (2011). The effect of augmented reality training on percutaneous needle placement in spinal facet joint injections. IEEE Transactions on Biomedical Engineering, 58 (7), pp. 2031-2037. 
Yong, L.A., Rivas, L.A. y Chaparro, J. (2010). Modelo de aceptación tecnológica (TAM): un estudio de la influencia de la cultura nacional y del perfil del usuario en el uso de las TIC. Innovar, 20, 36, pp. 187-204.

Yufeng, L. y Yamanaka, A. (2013). Exploring the effects of multimedia learning on pre-service teachers' perceived and actual learning performance. Educational Media International, 50 (4), 291-305. doi: 10.1080/09523987.2013.863556

\section{INFORMACIÓN SOBRE LOS AUTORES}

\section{Julio Barroso Osuna}

Universidad de Sevilla

Profesor Titular de la Universidad de Sevilla, adscrito a la Facultad de Ciencias de la Educación en el Departamento de Didáctica y Organización Educativa. Doctor en Ciencias de la Educación por la Universidad de Sevilla. Miembro del Grupo de Investigación Didáctica, miembro de EDUTEC. En la actualidad es director del Departamento de Didáctica y Organización Educativa. Cuenta con publicaciones tanto en grupo como en solitario relacionadas con la temática de las Nuevas Tecnologías aplicadas a la educación, así como su participación en diferentes cursos e investigaciones relacionadas con la misma temática.

\section{Julio Cabero Almenara}

Universidad de Sevilla

Catedrático de la Universidad de Sevilla, Director del Secretariado de Recursos Audiovisuales y Nuevas Tecnologías de la Universidad de Sevilla, Director del Grupo de Investigacón Didáctica (GID) de la Universidad de Sevilla. Ha impartido conferencias en diferentes Universidades españolas y extranjeras, sobre diferentes temáticas relacionadas con la Tecnología Educativa. Ha dirigido diferentes proyectos de investigación en planes competitivos, sobre el diseño de los vídeos, los entornos personales de aprendizaje, la realidad aumentada, los efectos cognitivos de las TIC. (http://grupodetecnologíaeducativa.es)

\section{(cc) BY-NO}

Los textos publicados en esta revista están sujetos a una licencia de Reconocimiento 4.0 España de Creative Commons. Puede copiarlos, distribuirlos, comunicarlos públicamente y hacer obras derivadas siempre que reconozca los créditos de las obras (autoría, nombre de la revista, institución editora) de la manera especificada por los autores o por la revista. La licencia completa se puede consultar en: Licencia Creative Commons Atribución-NoComercial-Compartir por igual 4.0 Internacional. 